\title{
Method of multi-sensor data Association based on large scale
}

\author{
LAN Xu-hui a , Li Ling-zhi, Guo Yong-ming \\ No.4 Department, Air Force Early Warning Academy, Wuhan 430019, China
}

\begin{abstract}
In the multi-sensor fusion system, great difference of detection information accuracy results high uncertainty of heterogeneous information correlation. This paper proposes a multi-sensor data correlation method basing on large scale which combines evidence theory and multi-factor fuzzy integrated decision theory in information correlation. For solving the problem of uncertainty of information correlation and obtaining evidence difficultly, the method first combines uncertain information evidence, and then obtains evidence from multi-factor fuzzy integrated decision membership degree function. The test results that heterogeneous information correlation using this method can conquer uncertainty of evidence combination and reduce the error and miss correlation rate.
\end{abstract}

\section{Introduction}

For a single sensor, it is difficult to detect the target correctly because of single detection means,limited data and failure detection probably. Multi-source heterogeneous data fusion can solve the problem. Multisensor heterogeneous data correlation is the base of multi-source data fusion.

Now, traditional data correlation algorithm is mainly based on statistics and fuzzy mathematics [1-2]. Weighting method, sequential method, dual-threshold, the nearest-neighbor method, K-NN method, and amended K-NN method are based on statistics. Fuzzy mathematics method, fuzzy dual-threshold method, fuzzy integrated function method and multi-factor fuzzy integrated decision method are based on fuzzy mathematics. Membership degree function of fuzzy mathematics can express fuzzy factors well, which brings high correlation rate[3]. These traditional methods are better for similar sensors data correlation. But there is high error correlation rate and miss correlation rate for uncertain heterogeneous data correlation.

This paper proposes a method of large scale for multisensor heterogeneous data correlation. Evidence theory that has preponderance for uncertain data, is used to multi-factor fuzzy integrated decision theory which can obtain evidence well from membership degree function[4]. Improved method step based on evidence theory and fuzzy decision theory is provided in this article. Simulation proves the method can reduce the error and miss correlation rate effectively for heterogeneous data correlation.

\section{Related theory}

\subsection{Multi-factor fuzzy integrated decision data correlation algorithm}

1) Determination of the fuzzy factors set

Taking Euclidian distances of target location and orientation angle of two different type of sensor to the factors set, that is fuzzy set $U=\left\{u_{k}\right\}=\left\{u_{1}, u_{2}, \cdots u_{n}\right\}$ with $n=2$. Where $u_{1}$ is the target location, $u_{2}$ is orientation angle, then:

$$
\left\{\begin{array}{l}
u_{1}(l)=\left[\left(x_{r}(l)-x_{a}(l)^{2}\right)+\left(y_{r}(l)-y_{a}(l)^{2}\right)\right]^{1 / 2} \\
u_{2}(l)=\left|\arctan \left[y_{r}(l) / x_{r}(l)\right]-\arctan \left[y_{a}(l) / x_{a}(l)\right]\right|
\end{array}\right.
$$

Where $u_{1}(l)$ is the diameter distance difference of two targets for time $l, u_{2}(l)$ is the orientation angle difference of two targets for time $l, x_{r}(l)$ is $\mathrm{X}$ location component of sensor 1 for time $l, x_{a}(l)$ is $\mathrm{X}$ location component of sensor 2 for time $l, y_{r}(l)$ is $\mathrm{Y}$ location component of sensor 1 for time $l, y_{a}(l)$ is $\mathrm{Y}$ location component of sensor 2 for time $l$. The right angle coordinate is used in calculation process.

2) Determination of the fuzzy judgments set

If the associated result of two sensors'data is divided into $m$ Level, the set consisted by these results is called the judgments set, and denoted $V=\left\{v_{l}\right\}=\left\{v_{1}, v_{2}, \cdots, v_{m}\right\}$, where $v_{l}, l=1,2, \cdots, m$ is the judgment results of $l$ th level. Any judgment result of two sensors' data is a fuzzy subset of $V$ actually.

We only concern about whether the target data association. Therefore, from the point of view of practical applications and simplifying the process, the judgments set is selected $V=\left\{v_{1}, v_{2}, \cdots, v_{m}\right\}$ with $\mathrm{m}=3$, where $v_{1}$ 
denotes correlation, $v_{2}$ denotes non-correlation, $v_{3}$ denotes uncertainty, that is $V=\{$ correlation, non-correlation, uncertainty $\}$. of course, the judgments set can be easier, that is $V=\{$ correlation, non-correlation $\}$. matrix

3) Determination of single factor fuzzy judgment

Single factor fuzzy judgment matrix is defined $R=\left(r_{k l}\right)_{n \times m}$ from $U$ to $V$ in direct product $\operatorname{set}_{U \times V}$, where $r_{k l}$ denoted the probability of the $l$ th result of two sensors' data when the $k$ th factor is congsidered.

According to the characteristics of fuzzy data correlation factors, memberships can be used with normal distribution, Cauchy distribution, middle distribution, etc. The paper selects normal distribution membership function. When $m=2$, the normal distribution membership degree based on the $k$ th factor judgment is:

$$
\begin{gathered}
r_{k 1}=\exp \left[-\tau_{k}\left(x^{2} / \sigma_{k}^{2}\right)\right] ; k=1,2 \\
r_{k 2}=1-\exp \left[-\tau_{k}\left(x^{2} / \sigma_{k}^{2}\right)\right] ; k=1,2
\end{gathered}
$$

Where $\tau_{k}$ is the adjustment coefficient; $\sigma_{k}^{2}$ is the variance. Therefore, the single factor fuzzy judgment matrix as follows:

$$
R=\left[\begin{array}{ll}
r_{11} & r_{12} \\
r_{21} & r_{22}
\end{array}\right]
$$

4) Criterion of multi-factor fuzzy judgment

The factor weight fuzzy set is $A=\left(a_{1}, a_{2}\right)$ with $a_{1}=0.7, a_{2}=0.3$ in the paper considering that distance precision is higher than orientation precision of sensor. Factor weight coefficients can be adjusted through a large number of experimental statistics.

Integrated decision of two sensors' data is compound effect of the factor weight fuzzy set A and fuzzy judgment matrix $\mathrm{R}$, which is the composition operation. The fuzzy set B of correlation probability of two sensors' data in $\mathrm{V}$ compositing by $\mathrm{A}$ and $\mathrm{R}$ is following:

$$
B=A \cdot R=\left(b_{l}\right)=\left(b_{1}, b_{2}\right)
$$

$b_{l}(l=1,2)$ is the membership degree of $v_{l}$ which is an element of judgments set. Composition operation has three kinds of algorithm, which are "weighting average", "subjective factor" and "hybrid". In order to equalize all factors based on weight, "weighting average" is used as follows:

$$
b_{l}=\sum_{k=1}^{2} a_{k} r_{k l}, l=1,2
$$

If data correlation probability of two sensors' for level $l$ is judged bythe maximum membership degree principle, the result is following:

$$
b_{l}=\max \left\{b_{1}, b_{2}\right\}
$$

However, the reliability is poor by above method. Because so long as the condition is $b_{1}>b_{2}$, the judgment is correlation. The composition operation is simple, but the result is not accurate.

\subsection{Evidence theory}

D-S evidence theory defined a space $\Theta$ which is called frame of discernment. It is composed of mutual exclusive and exhaustive elements. Any proposition A of the problem domain is belonged to powers set $2^{\Theta}$. Basic probability assignment function is defined in $2^{\Theta}$ set: $m: 2^{\Theta} \rightarrow[0,1] . \mathrm{m}$ satisfies three conditions, which are: (1) $m(\Theta)=0 ;(2) 0 \leq m(A) \leq 1 ;(3) \sum_{A \subset \Theta} m(A)=1$.

$m(A)$ denotes a true subset which supports proposition A by evidence but not any $\mathrm{A}$. If $\mathrm{A}$ is a subset of $\Theta$, and satisfies $m(A)>0$, it is called focus element of evidence. Interval $[\operatorname{Bel}(A), \operatorname{Pl}(A)]$ is used to describe event A in evidence theory, where Bel is called belief function and $P l$ is called plausibility function.

D-S evidence theory provides a very useful composition formula. It can compose evidences from multiple evidence sources. The formula is defined as follows (an example for two evidences):

$$
m(A)=\frac{1}{1-K} \sum_{A_{i} \cap B_{j}=A} M_{1}\left(A_{i}\right) M_{2}\left(B_{j}\right)
$$

With $k=\sum_{A_{i} \cap B_{j}=\Phi} M_{1}\left(A_{i}\right) M_{2}\left(B_{j}\right)$.Its value reflects conflict degree of evidence. The coefficient $1 /(1-k)$ is called normalization factor. Its role is to avoid that nonzero probability is assigned to the null set when composing evidences.

\section{Improved correlation method based on evidence theory}

Frame of discernment for evidence is $\Theta=$ (correlation, non-correlation, uncertainty) .The factors set influencing the decision is $U=\left\{u_{1}, u_{2}, \cdots u_{n}\right\}$. Supposing that the basic probability of the main two factors influencing data correlation is assigned as following:

$m_{1}($ correlation $)=0.6 m_{1}($ non-correlation $)=0.3$

$m_{1}$ (uncertainty) $=0.1$

$m_{2}($ correlation $)=0.4 m_{2}($ non-correlation $)=0.5$

\begin{tabular}{|c|c|c|c|}
\hline \multirow[b]{2}{*}{$\begin{array}{c}\text { Assignment } \\
\qquad m_{2}\end{array}$} & \multicolumn{3}{|c|}{ Evidence synthesis calculated values } \\
\hline & $\begin{array}{l}m_{1}(\text { correlation }) \\
0.6\end{array}$ & $\begin{array}{c}m_{1}(\text { non- } \\
\text { correlation }) \\
0.3\end{array}$ & $\begin{array}{c}m_{1} \text { (uncertainty) } \\
0.1\end{array}$ \\
\hline $\begin{array}{c}m_{2} \text { (correlation) } \\
0.4\end{array}$ & 0.24 & 0.12 & 0.04 \\
\hline $\begin{array}{c}m_{2} \text { (non- } \\
\text { correlation) } \\
0.5 \\
\end{array}$ & 0.3 & 0.15 & 0.05 \\
\hline $\begin{array}{c}m_{2} \text { (uncertainty) } \\
0.1\end{array}$ & 0.06 & 0.03 & 0.01 \\
\hline
\end{tabular}

$m_{2}$ (uncertainty) $=0.1$

The basic probability is obtained by membership degree functions of multi-factor fuzzy integrated decision data association algorithm.

Synthesizing evidence by evidence theory:

Table 1. calculation of evidence synthesis 
correlation $)+m_{1}$ (correlation) $\times m_{2}$ (uncertainty $)+$ $m_{1}$ (non-correlation $) \times m_{2}($ uncertainty $)=0.4$

Integrated probability after evidence synthesis is assigned:

$m($ correlation $)=0.6 m($ non-correlation $)=0.375$

$m$ (uncertainty $)=0.025$

If data correction decision rules are:

Table 2. Data Association guidelines

\begin{tabular}{|l|c|}
\hline \multicolumn{1}{|c|}{ Decision } & Rules \\
\hline correction & $\begin{array}{c}m \text { (correlation) }>m(\text { non-correlation }) \text { and } \\
m(\text { correlation })>m(\text { uncertainty })\end{array}$ \\
\hline non-correction & $\begin{array}{c}m \text { (non-correlation }) \geq m(\text { correlation }) \text { and } \\
m(\text { non-correlation }) \geq m(\text { uncertainty })\end{array}$ \\
\hline Uncertainty & $\begin{array}{l}m \text { (uncertainty })>m \text { (correlation) and } \\
m(\text { uncertainty })>m(\text { non-correlation })\end{array}$ \\
\hline
\end{tabular}

By combination rules of evidence, the result is data correction.

\section{Simulation analysis}

$\Theta=\{A, B, \theta\}$,Initial evidence formed by decision

factor that two weights are 0.7 and 0.3 is:

$$
\begin{array}{llll}
a_{1}(\text { weit })=0.7 & m_{1}(\mathrm{~A})=0.7 & m_{1}(\mathrm{~B})=0.3 & m_{1}(\theta)=1-a_{1}=0.3 \\
a_{2}(\text { weit })=0.3 & m_{2}(\mathrm{~A})=0.1 & m_{2}(\mathrm{~B})=0.9 & m_{2}(\theta)=1-a_{1}=0.7
\end{array}
$$

Evidences are obtained by multi-factor fuzzy integrated decision method. The result using multi-factor fuzzy integrated decision membership degree synthesis data correction algorithm is following

$$
\begin{gathered}
\left\{\begin{array}{l}
m(A)=a_{1} m_{1}(A)+a_{2} m_{2}(A)=0.52 \\
m(B)=a_{1} m_{1}(B)+a_{2} m_{2}(B)=0.48 \\
m(\theta)=m_{1}(\theta)+m_{2}(\theta)=1
\end{array}\right. \\
\quad \text { After normalization: }
\end{gathered}
$$

The evidences combination result is that

$$
\left\{\begin{array}{l}
m(A)=m_{1} \oplus m_{2}(A)=0.23 \\
m(B)=m_{1} \oplus m_{2}(B)=0.31 \\
m(\theta)=m_{1} \oplus m_{2}(\theta)=0.46
\end{array}\right.
$$

Data correction algorithm using multi-factor fuzzy integrated decision, although can choose A as the final decision according to new evidences. Obviously, the trust degree difference for $\mathrm{A}$ and $\mathrm{B}$ is only 0.02 , but uncertainty degree is 0.5 , that is unreasonable and is not conformity with algorithm rule. Using evidences combination, the trust degree difference for $\mathrm{A}$ and $\mathrm{B}$ is 0.08 , but uncertainty degree reduces to 0.046 , and the result is $\mathrm{B}$. Therefore data correction algorithm using evidence theory can reduce uncertainty degree effectively, obtain better reasonable correction result, and reduce error correction rate.

\section{Conclusions}

Multisource heterogeneous data correction exits high uncertainty degree, but evidence theory can depose the problem well. It is difficult to obtain evidence, but it is easy to obtain evidences by multi-factor fuzzy integrated decision membership degree function. Therefore, for high uncertainty degree of heterogeneous data correction, combining evidence theory and multi-factor fuzzy integrated decision can reduce error correction rate and leak correction rate. In addition, evidence theory gets some beneficial progress in disposing evidence conflict, calculation complexity and evidence combination explosion etc. The dependability of evidence theory becomes better in engineering. As a result, it is a useful expand that evidence theory is used to heterogeneous data correction.

\section{References}

1. he you, Wang Guohong, Guan Hin .. The theory of information fusion and its application [M]. Beijing : Publishing House of electronics industry ,2010:178.

2. Han Chongzhao, Zhu Hongyan, overcome . Source new information fusion [M]. Beijing : Tsinghua University Press , 2006: 290.

3. he you, land, Peng Yingning, and so on . Tracks correlation algorithm based on fuzzy synthetic function $[\mathrm{J}]$. Journal of Naval Engineering College ,1999,89 (4):1-11

4. Dezert J. Foundations for a New Theory of Plausible and Paradoxical Reasoning, Information \& Security [J]. An Int Journal, 2002, 45 (9):13-57.

5. Dezert J, Smarandache F. Advances and Applications of DSmT for Information Fusion [M]. American Research Press, Rehoboth, 2009:15-20.

6. Wei-Ping Huang, $\mathrm{Xu} \mathrm{Yu}$, Wang Jie . Comprehensive utilization of various information of / Data Association of radar [J]. Chinese Journal of sensors ,2009,22(6) : 216-821.

7. Dong Hongbo, busy hero Liu Jin, Zhang Chunmei , and so on . Research on airborne fuzzy track correlation algorithm with dissimilar sensors [J]. Sensors and Microsystems ,2010,29 ( 7 ): 8-10.

8. Chen Shiyou, xiaohou, Liu Hao . Representation of uncertainty in track correlation [J]. Electronics ,2011,39 (7) : 1589-1593.

9. A. Mecke, I. Lee, J.R. Baker jr., M.M. Banaszak Holl, B.G. Orr, Eur. Phys. J. E 14, 7 (2004)

10. M. Ben Rabha, M.F. Boujmil, M. Saadoun, B. Bessaïs, Eur. Phys. J. Appl. Phys. (to be published) 